\title{
AAAI: an Argument Against Artificial Intelligence
}

\author{
Sander Beckers
}

\begin{abstract}
The ethical concerns regarding the successful development of an Artificial Intelligence have received a lot of attention lately. The idea is that even if we have good reason to believe that it is very unlikely, the mere possibility of an AI causing extreme human suffering is important enough to warrant serious consideration. Others look at this problem from the opposite perspective, namely that of the AI itself. Here the idea is that even if we have good reason to believe that it is very unlikely, the mere possibility of humanity causing extreme suffering to an AI is important enough to warrant serious consideration. This paper starts from the observation that both concerns rely on problematic philosophical assumptions. Rather than tackling these assumptions directly, it proceeds to present an argument that if one takes these assumptions seriously, then one has a moral obligation to advocate for a ban on the development of a conscious AI.
\end{abstract}

\section{Introduction}

In the wake of the recent boom in the field of Artificial Intelligence, there has been an equally spectacular boom in apocalyptic predictions regarding $\mathrm{AI}$ and the faith of mankind. Extrapolating the accelerating progress of AI and our dependence on it, doomsayers worry that it is only a matter of time before we develop an Artificial General Intelligence, or Strong AI, which would be so powerful that it could cause terrible global suffering and possibly even the extinction of our species (Bostrom, 2014; Hawking, 2014; Hawking, Russell, Tegmark, \& Wilczek, 2014; Musk, 2015; Tegmark, 2015). In fact, our situation is deemed so worrisome, that several new research centers have been created with the explicit aim of reducing the potential dangers of AI. ${ }^{1}$

Sander Beckers

Utrecht University, Department of Philosophy and Religious Studies

e-mail: srekcebrednas@gmail.com

WWW home page: https://sanderbeckers.com

${ }^{1}$ The Center for Human-Compatible AI, the Machine Intelligence Research Institute, OpenAI, the Future of Humanity Institute, and the Foundational Research Institute, to name just a few. Of course these institutes do not focus exclusively on the long-term existential risks posed by AI, but also on the abundant more concrete risks that current AI already poses. 
Some authors have also turned the table on the ethical concerns regarding AI. Instead of merely considering the harm that an AI could bring upon humans, they also consider the harm that could be brought upon an AI by humans (Bostrom, 2014; Mannino et al., 2015; Metzinger, 2010; Sotala \& Gloor, 2017). The idea is that a truly intelligent AI would also develop consciousness, and with consciousness comes the capacity for emotions, agency, and all other aspects that we associate with subjects that deserve moral consideration (Bostrom, 2014; D. J. Chalmers, 1996; Dennett, 1993; Metzinger, 2010). For example, one could argue that the continued development of AI as systems that are entirely subjected to our every wish and command would amount to re-introducing slavery (Walker, 2006).

Ironically, the strong pessimism towards the future prevalent in both types of ethical concern mentioned above is founded on underlying assumptions that reveal a strong optimism towards the present: the assumption that the current rate of progress within $\mathrm{AI}$ is bound to continue unabated and the assumption that we have a clear understanding of certain deep philosophical issues. We set aside entirely whether the former assumption is justified, as that is something to be settled by a technical scientific discussion. Instead, the focus of this paper is on the latter more philosophical assumptions.

Concretely, the first type of ethical concern is based on the assumption that an AI could become superintelligent and the second type of ethical concern is based on the assumption that an AI could suffer. Both assumptions are highly controversial from a philosophical perspective. Firstly, it is not at all clear whether the very notion of superintelligence makes any sense, especially as it concerns non-human entities. Secondly, it is undoubtedly an understatement to say that we do not yet have a good understanding of how consciousness arises in human beings, let alone elsewhere.

The goal of this paper is not to call into question these assumptions directly. Instead, the aim is to show that if we actually take them seriously, then humanity has a moral obligation not to create a conscious AI. If this argument is successful, researchers in AI who are reluctant to accept its conclusion will be pressed with the challenge of either dropping one - or both - of these assumptions, or do some soul-searching and become advocates of a ban on the creation of a conscious AI.

In addition to said assumptions, the argument here developed also assumes a minimal utilitarian outlook. That is, it is assumed that utilities appropriately capture certain quantifiable and objective features of our moral framework, and that all else being equal, we have a moral duty to create more utility rather than less. What makes this outlook minimal, is that it leaves open entirely whether utilities capture all morally salient features.

\section{Supersuffering}

The first assumption we encountered above is that an AI could develop superintelligence, which is a level of intelligence that far exceeds our own human intelligence (Bostrom, 2014; D. Chalmers, 2010). In the words of Bostrom (2006), "we mean an 
intellect that is much smarter than the best human brains in practically every field, including scientific creativity, general wisdom and social skills". Although it is quite straightforward to imagine an AI that outperforms human beings in computationally demanding tasks, it is much harder to conceive of an intellect that is much smarter than human beings in, say, social skills. Presumably we are talking about the skills required to socialize with human beings, after all.

More generally, given that the concept of intelligence was constructed to capture a property of human beings, it is not at all clear what it even means to surpass $h u$ man intelligence. The way in which the idea of superintelligence is invoked implies that it isn't merely a matter of being able to think faster than a human or having a larger memory capacity, for if that were the case then humans would still be able to outsmart it by using their collective intelligence together with computers and other technology. On the contrary, the type of superintelligence produced by a singularity, or one that has the capacity to subdue and potentially destroy all of mankind, is such that it would be able to gain insights that are seemingly forever beyond our grasp. Here one is inclined to paraphrase Wittgenstein: "If an AI could speak, we could not understand it".

Without a proper theory of intelligence that enables us to make sense of the idea that intelligence comes in degrees which extend far beyond the range of anything we find in humans, we ought to split up our single assumption into several separate assumptions that make explicit what the idea of a superintelligent $\mathrm{AI}$ requires.

Assumption 1 There exist quantifiable, mental, and human properties that an AI can have.

Assumption 2 If an AI can have a quantifiable, mental, and human property, then it can have this property to a degree which extends far beyond the human level.

Assumption 3 Intelligence is one such property for which Assumption 1 holds.

Taken together, these assumptions allow us to conclude that a future AI could be superintelligent.

The second assumption mentioned earlier is that an AI could suffer. Given that, like intelligence, suffering comes in degrees, we can rephrase this assumption as follows:

Assumption 4 Suffering is one such property for which Assumption 1 holds.

As with intelligence, we can apply Assumption 2 to conclude that a future AI could supersuffer, i.e., it could suffer to a degree that far exceeds any potential human suffering.

One might object that Assumption 2 is too strong, for the idea of superintelligence only requires an assumption of that form to hold for intelligence. Yet without a theory of intelligence that gives us this particular assumption, restricting Assumption 2 to intelligence would be gratuitous: we have no grounds whatsoever for stipulating that there is something peculiar about intelligence as compared to suffering so that it is the only candidate for amplification to a super-level.

\footnotetext{
2 The original mentions a lion, rather than an AI. (Wittgenstein, 1953, p.223)
} 
In fact, given Assumptions 1, 3 and 4, the concept of supersuffering seems far less problematic than the concept of superintelligence once we shift focus from a single agent to a group of agents. ${ }^{3}$ The reason is that more agents suffering to some degree $X$ always implies more total suffering than less agents suffering to the same degree $X$, whereas the same does not hold for intelligence. In other words, suffering is far more cumulative than intelligence. For example, except for the speed at which they can solve certain problems, two identical agents need not be any more intelligent overall than each agent considered separately. However, if two identical agents are being tortured then there is clearly a lot more suffering than when only of of them is being tortured.

Further, we find additional support for the possibility of a supersuffering AI from other sources. Sotala and Gloor (2017) offer a detailed analysis of the potential suffering that could be caused by an AI. While they focus mostly on human suffering, they also mention that "these [future technologies] may enable the creation of mind states that are worse than the current biopsychological limits." They provide interesting thought experiments to substantiate this claim. In a similar vein, Metzinger (2013) states that future AIs "might suffer emotionally in degrees of intensity or in qualitative ways completely alien to us that we, their creators, could not even imagine."

Such a supersuffering AI would amount to what can be called a negative utility monster: a being whose utility is so incredibly low that all of our efforts should go to increasing its utility, instead of wasting energy on increasing the comparatively negligible utilities that we human beings could obtain. The notion of a positive utility monster was posited by Nozick in order to highlight a counterintuitive consequence of utilitarianism (Nozick, 1974, p. 41):

Utilitarian theory is embarrassed by the possibility of utility monsters who get enormously greater sums of utility from any sacrifice of others than these others lose ... the theory seems to require that we all be sacrificed in the monster's maw, in order to increase total utility.

One standard utilitarian reply is to object that such a monster is not conceivable, for no single entity could possibly have such large quantities of utility, be it negative or positive (Parfit, 1984). Note that our starting point, however, contains the observation that the recent success of AI has dramatically altered the type of entities that people claim they can conceive of. So if by now we can conceive of an AI as an intelligence monster, and we can conceive of an AI as having morally salient mental states such as suffering, then the mere claim that we cannot conceive of an AI as a negative utility monster does not carry much weight.

Other utilitarians are indeed prepared to bite the bullet and concede that such a monster would have to be the primary target of moral concern. For example, Singer (2009) says that "if we ever encountered Martians who could convince us that they had a vastly greater capacity for happiness than we do, then it could be a problem."

So far we have focussed on the suffering of a single AI, but the problem of supersuffering becomes all the more pressing once we aggregate the suffering of multiple

${ }^{3}$ In the case of AI systems, it might even be that a group of agents could easily merge into a single agent. For sake of simplicity, we leave this speculative possibility aside. 
AI systems, over extended periods of time. Once we are able to create an AI - in the sense of a superintelligent and conscious AI as we have been considering - it is reasonable to assume that we go ahead and produce a large number of copies. From there it is only a small step to imagining horrible scenarios in which there would be more artificial suffering than all human suffering, past and present, combined.

For example, say we are able to create a holographic AI that is the result of uploading a person's brain and running it as a hologram that looks like the person. Creative as human beings are, a cunning investor uses this technology to construct a profitable attraction: for a couple of dollars, visitors get to pull the switch on a holographic electric chair in which is seated a holographic copy of a convicted murderer whose original human version has long since been executed by an actual electric chair. The fact that the hologram experiences the exact same excruciating pain makes the attraction widely successful. Millions of visitors come to pull the switch, causing millions of holograms to suffer terribly. To top it all off, each visitor receives a keychain containing a copy of the hologram that is continuously, during every single second of the day, year after year, experiencing this execution. Unlike an actual human being, these holographic AIs do not have the benefit of death to put their suffering to an end. Obviously this scenario is extremely far-fetched, but given our earlier assumptions, it is certainly conceivable. This is confirmed by millions of viewers of the superb sci-fi television series Black Mirror, in which this very scenario is enacted (and others like it).

For another illustration, one could imagine that the experience of empathy is achieved in an AI by automatically replicating any suffering that it observes. A reason for programming the AI in this manner is that it might very well be a good way to ensure that an $\mathrm{AI}$ is highly sensitive to, and aware of, any form of human suffering - which it better be if we expect it to avoid treating humans as mere instruments for attaining its objectives. Now imagine that such an AI has access to all of recorded human history. In particular, it can immediately access all audio-visual material ever produced. Further, the AI is so fast and unbounded in resources that for every single decision it makes, it takes into account the total amount of evidence which is available to it. Say it makes a million decisions per second. This implies that during a single second, a single AI goes through the entire amount of suffering ever recorded a million times over. The fact that all of this happens within a single second should not be seen as a mitigating factor, for according to Bostrom and Yudowsky's plausible principle of the subjective rate of time, "In cases where the duration of an experience is of basic normative significance, it is the experience's subjective duration that counts." (2014, p. 326). Given the speed at which we can expect an AI to be operating, this principle in and of itself is already sufficient to guarantee that the experience of suffering for an $\mathrm{AI}$ can take on far more extreme forms than it can for human beings: a single experiment that goes astray for a few seconds could result in an AI suffering for many years.

One might counter that we can avoid such scenarios by implementing policies that forbid them. But such policies would be unable to prevent similar scenarios in which the suffering is unintended, and worse even, scenarios in which the suffering goes by entirely unnoticed. Once an AI has the capacity to suffer, then all it would 
take is some bug in the code for similar scenarios to unfold. For example, imagine that there is some complicated version of the millennium bug, which is activated in billions of AIs at the same time and causes them to suffer to the astronomic extend portrayed above before we even know what is going on.

Metzinger (2013) also focusses on this issue, highlighting the "possibility that non-biological subjects of experience have already begun to suffer before we as their human creators have even become aware of this fact." He develops a theory that allows for the quantification of suffering, and posits that it is our duty to minimize the frequency of conscious experiences that involve suffering (Metzinger, 2017). As a consequence, he concludes that we should ban the development of an AI, in the strong sense of AI as we are using it, stating the following principle (2013, p. 3):

We should not deliberately create or even risk the emergence of conscious suffering in

artificial or postbiotic agents, unless we have good reasons to do so.

Mannino et. al. (2015) reach a similar conclusion in their overview of the moral risks posed by the development of AI, stating that "the (unexpected) creation of sentient artificial life should be avoided or delayed wherever possible, as the AIs in question could - once created - be rapidly duplicated on a vast scale."

Given the assumptions made at the outset, and the severity of the sketched scenarios, the only way to avoid accepting these negative verdicts is to follow through on Metzinger's hint and offer good reasons as to why the possibility of supersuffering is an acceptable price to pay. Three straightforward suggestions present themselves as plausible candidates:

1. The attempt at creating an $\mathrm{AI}$ is not at all special in this regard, since all other acts that we perform as humanity today also run the risk of causing extreme suffering in the future, and nevertheless we find this perfectly acceptable.

2. The negative scenario of supersuffering is compensated by a positive scenario of an AI experiencing superpleasure.

3. The expected benefits for mankind that come from creating an AI outweigh the possibility of supersuffering.

In the remainder of this paper the aim is to show why all three suggestions fail.

\section{The Unique Risk of Creating an AI}

In order to show how the consequences of creating an AI are unlike the consequences of other acts that we collectively engage in, we make explicit in what manner the risk of an $\mathrm{AI}$ undergoing extreme suffering is unique. We do so by comparing strategies that humanity could adopt in order to avoid risking human suffering with strategies that humanity could adopt in order to avoid risking AI suffering.

A first thing to note is that there exists one very radical strategy that humanity could adopt in order to avoid any risk of human suffering in the long term, namely to stop having children altogether. We here take it for granted that this strategy is morally unacceptable, without offering further motivation. 
Principle 1 (Acceptable) A strategy is acceptable unless it certainly causes the extinction of mankind.

The only reason we mention this principle is for sake of completeness, since in recent years Benatar (2006) has championed the extremely controversial position of anti-natalism, according to which it is immoral to have children. This position is based on negative utilitarianism, a topic to which we come back later.

Now we turn to strategies that are acceptable and ensure the avoidance of some terrible outcome.

Definition 1 (Avoidable). A possible outcome is certainly avoidable if there exists an acceptable strategy that certainly prevents the outcome of occurring.

We already concluded that humanity might cause future AIs to experience supersuffering. In order to invoke the above definition, we need to add the following trivial counterpart.

Premise 1 If all of humanity does not attempt to create an AI, then certainly there will never be an AI that experiences supersuffering.

This leads us to conclude the following:

Conclusion 1 A supersuffering AI is certainly avoidable.

On the short term, and when considering a single agent, there are many negative outcomes which are certainly avoidable. This no longer holds if we consider all of humanity and extend our horizon into the far future: given our limited knowledge of the world, and the almost infinite complexity of the causal chain that results from our actions, we are ignorant with respect to the long-term consequences of our actions on the well-being of humanity.

Premise 2 There exists a time $t$ such that no matter what acceptable strategy we adopt, to the best of our knowledge, it is possible that this strategy causes extreme human suffering after $t$.

At first glance there appear to be many strategies that defy the above premise. For example, think of our efforts to cure cancer. Either these are successful, in which case they would prevent a great deal of suffering, or they would be unsuccessful and not have any impact at all. But this analysis only considers the most likely outcomes that each alternative would have. Although unlikely, it is definitely possible that our efforts to cure cancer result in the creation of a deadly and contagious virus, which causes many more deaths than the disease which it was supposed to cure. Or it is possible that by curing cancer, the next would-be genocidal dictator is kept alive, and therefore able to live out his evil intentions.

We can now apply Definition 1 to reach the following conclusion:

Conclusion 2 Extreme human suffering is not certainly avoidable. 
This conclusion provides us with a distinguishing feature of the risk that comes with the attempt to create a conscious AI. Any suffering that a future AI might experience is certainly avoidable, whereas this does not hold for the suffering of future humans. Therefore the first suggestion does not succeed in giving us a good reason why we should risk a supersuffering AI.

\section{Moral Asymmetry}

At this point we can draw the following worrisome conclusion.

Conclusion 3 It is possible that by creating an AI, we will cause a unique and extreme form of suffering that could certainly have been avoided.

Still, an optimist might argue, completely analogous to this depressing conclusion, it is also possible that by creating an AI we will cause a unique and extreme form of pleasure that would otherwise certainly have been avoided. Hence the route for the second suggestion to defend our attempt at creating an AI is still open.

However, there is a strong intuition that is so well-embedded in our everyday life that only an extreme utilitarian would object to it: it is more important to avoid suffering than it is to create pleasure. Moore (1903) was the first to express this intuition, but Popper was its most famous defender (Popper, 1945):

We should realize that from a moral point of view suffering and happiness must not be treated as symmetrical; that is to say the promotion of happiness is in any case much less urgent than the rendering of help to those who suffer, and the attempt to prevent suffering.

This idea forms the basis of "moderate negative utilitarianism", which considers it our primary duty to avoid suffering (Chauvier, 2014; Mayerfeld, 1999; Metzinger, 2013; Parfit, 1997). The asymmetry between pleasure and pain that lies at its core is evident in the medical principle "first do no harm", and is confirmed by the moral risk-aversion that is widespread in our behaviour. ${ }^{4}$

For example, assume you may press a button such that with probability 0.5 a random person's leg will be broken, and with probability 0.5 someone's broken leg will be healed, and neither person has any say in the matter. Or imagine that if you press the button, a random person will be hit in the face, but offered a massage afterwards. It goes without saying that it is immoral to press the button.

Further, this asymmetry increases as the intensity of the suffering and pleasure increases. For example, if someone insults you but then offers a compliment, you probably will not have hard feelings towards that person. But if they torture you, it is hard to imagine what form of pleasure they could offer you to avoid feeling terribly wronged by that person. In fact, some even go so far as to state that certain amounts of suffering cannot be compensated by any amount of pleasure at all, a position Hurka (2010) describes as the limit asymmetry thesis: "There is some intensity $n$ such that a pain of intensity $n$ is more evil than any pleasure could be good."

\footnotetext{
${ }^{4}$ See the papers cited above for many more interesting examples.
} 
In light of all this, the following moral principle is endorsed by a broad range of ethical positions and has a prima facie intuitive appeal:

Principle 2 (Moral asymmetry) All else being equal, the moral blameworthiness for causing a degree of suffering $X$ is greater than the moral praiseworthiness for causing a degree of pleasure X. Further, the difference between the degree of blame and praise strictly increases with $X$.

Nevertheless, as said, a strict utilitarian could insist on the symmetry between pleasure and suffering, and hence reject this principle. In that case, the possibility of a supersuffering AI could be compensated, on the condition that the probability of superpleasure is significantly greater than that of supersuffering.

Setting this caveat aside, we conclude that our expected blameworthiness when continuing the development of $\mathrm{AI}$ is higher than when we stop all research on AI, and hence the second candidate suggested as a good reason for risking supersuffering is ruled out as well.

\subsection{Anti-natalism}

As promised, we briefly return to the position of anti-natalism. We rejected this position outright because of the simple fact that almost everyone would find the prospect of mankind going extinct quite depressing, to say the least (whereas few would mourn the non-existence of sentient AIs). We now clarify how this position relates to the one here developed.

We motivated Principle 2 by reference to moderate negative utilitarianism. By using the label "moderate", its proponents wish to distance themselves from "negative utilitarianism", which embraces the far stronger claim that even the slightest amount of suffering can never be compensated by any amount of pleasure whatsoever. Given that every human being will experience some amount of suffering throughout its life, this claim implies that it is immoral to bring children into existence, no matter what the circumstances.

In contrast, a moderate negative utilitarian can perfectly well defend having children, on grounds of the fact that most people end up leading lives which have an acceptable amount of suffering compared to the amount of pleasure. In other words, most people end up leading lives worth living. Only if it were very likely that one's child would experience constant suffering would it follow that it is better not to have a child, a conclusion which most people would fully endorse. ${ }^{5}$

Further, even if we were to assume that the probability of extreme suffering for a child is identical to that of supersuffering for an AI, the astronomical difference in

\footnotetext{
${ }^{5}$ Metzinger also makes this point, and adds that anti-natalism regarding artificial life is far more plausible than its biological counterpart (Metzinger, 2013, 2017). To avoid unnecessary complication, we make clear that we need not get into the issue of abortion, but are talking simply about preventing the act of human fertilization in the first place.
} 
the degree of suffering involved suffices to separate the application of Principle 2 to the latter from its application to the former.

\section{The Ethical Priority of Artificial Suffering}

The third suggestion that might offer a good reason for going ahead and risk the prospect of AI systems supersuffering, is that this would be an acceptable price to pay given the expected benefits for mankind that would follow the invention of truly intelligent AI. That we consider this candidate suggestion last is due to the simple reason that the discussion of the previous two suggestions already puts considerable pressure on this idea.

First, so far we have completely ignored the first ethical concern mentioned at the outset, namely the concern that the creation of an AI could cause extreme human suffering. If we take seriously the doomsayers, meaning we attribute a small but non-negligible probability to the type of worst-case scenarios in which AIs will wreck havoc and destruction everywhere, then we have every reason to believe that the overall expected benefits for mankind in the long run are drastically negative.

Second, even without invoking these worst-case scenarios, given the myriad degrees of freedom that the organization of human society possesses, there is no reason to assume that AI is in any way necessary for human beings to flourish in the long run. For all we know, there exist strategies not involving the creation of AI that would cause an even higher increase in human pleasure after the time horizon $t$ mentioned in Conclusion 2 than an AI could ever produce. In sum, as per Conclusion 1, the creation of an AI would result in us giving up on the certainly avoidable outcome of a supersuffering AI, whereas it is by no means clear how the creation of an AI relates to the prospects of mankind after time $t$.

Third, we can combine the asymmetry between suffering and pleasure captured by Principle 2 with the astronomical difference in orders of magnitude between the amount of suffering depicted in the scenarios from Section 2 and any feasible amount of human pleasure that could occur before time $t$ mentioned above to see that the benefits for mankind before time $t$ do not even come close to compensating for a supersuffering AI. That is, they do not come close if we accept the following plausible principle:

Principle 3 (Non-discrimination) When evaluating the overall expected benefits of creating an AI, we ought not discriminate between the suffering/pleasure of an AI and a human being. ${ }^{6}$

When reformulated in terms of different groups of human beings, this principle is a bedrock of any modern moral system, and hence it hard to see how it could fail to apply when we extend it to other conscious beings that have an even stronger capacity for suffering and pleasure than humans do. Singer (2011, p. 50) puts it thus:

${ }^{6}$ This principle could also be generalized to include certain animals. However, one would first have to introduce Assumptions 1 and 4 reformulated for animals. 
If a being suffers, there can be no moral justification for refusing to take that suffering into consideration. No matter what the nature of the being, the principle of equality requires that the suffering be counted equally with the like suffering - in so far as rough comparisons can be made - of any other being.

In sum, the combination of these two arguments blocks the last suggested candidate:

Conclusion 4 From an ethical point of view, the possibility for an AI to experience supersuffering takes precedence over the expected benefits that an AI will produce for mankind.

\section{Conclusion}

In Section 2 we examined the basis for two popular and controversial assumptions regarding Artificial Intelligence, and argued that accepting these assumptions leads to the moral principle that we should not create a conscious AI, unless we can offer good reasons to do so. In the subsequent sections we rejected three natural candidates for such reasons. Therefore, if we take seriously our two initial assumptions, we are forced to accept the following conclusion:

Conclusion 5 Humanity should not attempt to create a conscious AI. $^{7}$

Given the gravity of this conclusion, it is incumbent upon each and every AI researcher to closely inspect said assumptions, and make a choice: either refrain from endorsing both of them and explain why, or advocate for a ban on the creation of a conscious AI.

Acknowledgements The author gratefully acknowledges financial support from the ERC-2013CoG project REINS, nr. 616512.

\section{References}

Benatar, D. (2006). Better never to have been. Oxford University Press.

Bostrom, N. (2006). How long before superintelligence? Linguistic and Philosophical Investigations, 5(1), 11-30.

Bostrom, N. (2014). Superintelligence: Paths, dangers, strategies. Oxford University Press.

\footnotetext{
${ }^{7}$ Humanity here refers to current humanity. If at some point in the future we discover a method of working on the development of a conscious AI that is certain to avoid supersuffering, then obviously this conclusion could be retracted. For now, however, we are just as far removed from the discovery of such a method as we are of the discovery of a conscious AI itself.
} 
Bostrom, N., \& Yudowsky, E. (2014). The cambridge handbook of artificial intelligence. In K. Frankish \& W. M. Ramsey (Eds.), (chap. The Ethics of Artificial Intelligence). Cambridge University Press.

Chalmers, D. (2010). The singularity: A philosophical analysis. Journal of Consciousness Studies, 17(9-10), 7-65.

Chalmers, D. J. (1996). The conscious mind: In search of a fundamental theory. Oxford University Press.

Chauvier, S. (2014). A challenge for moral rationalism: why is our common sense morality asymmetric? In J. Dutant, D. Fassio, \& A. Meyan (Eds.), Liber amicorum pascal engel (p. 892-906). University of Geneva.

Dennett, D. C. (1993). Consciousness explained. Penguin UK.

Hawking, S. (2014). Stephen hawking warns artificial intelligence could end mankind. Retrieved from http://www. bbc.com/news/technology $-30290540$

Hawking, S., Russell, S., Tegmark, M., \& Wilczek, F. (2014). Retrieved 14 April 2018, from https://www.huffingtonpost.com/stephen -hawking/artificial-intelligence_b_5174265.html

Hurka, T. (2010). Asymmetries in value. Nous, 44(2), 199-223.

Mannino, A., Althaus, D., Erhardt, J., Gloor, L., Hutter, A., \& Metzinger, T. (2015). Artificial intelligence: Opportunities and risks. Policy Paper by the Effective Altruism Foundation, 2, 1-16.

Mayerfeld, J. (1999). Suffering and moral responsibility. Oxford University Press.

Metzinger, T. (2010). The ego tunnel: The science of the mind and the myth of the self. New York: Basic Books.

Metzinger, T. (2013). Two principles for robot ethics. In E. Hilgendorf \& J.P. Günther (Eds.), Robotik und gesetzgebung (p. 263-302). Baden-Baden: Nomos.

Metzinger, T. (2017). Suffering. In K. Almqvist \& A. Haag (Eds.), The return of consciousness. Axess Publishing.

Moore, G. (1903). Principia ethica. Cambridge University Press.

Musk, E. (2015). Retrieved 14 Apr 2018, from https://www. theguardian .com/technology/2015/jan/16/elon-musk-donates-10m -to-artificial-intelligence-research

Nozick, R. (1974). Anarchy, state, and utopia. Basic Books.

Parfit, D. (1984). Reasons and persons. Oxford University Press.

Parfit, D. (1997). Equality and priority. Ratio, 10(3), 202-221.

Popper, K. (1945). The open society and its enemies, vol. $i$. Routledge.

Singer, P. (2009). Back talk: Peter singer. Retrieved 14 Apr 2018, from https : / / www.thenation.com/article/back-talk-peter-singer/

Singer, P. (2011). Practical ethics. Cambridge University Press.

Sotala, K., \& Gloor, L. (2017). Superintelligence as a cause or cure for risks of astronomical suffering. Informatica, 41, 389-400.

Tegmark, M. (2015). An open letter: Research priorities for robust and beneficial artificial intelligence. Retrieved 14 Apr 2018, from https:// futureoflife.org/ai-open-letter 
Walker, M. (2006). A moral paradox in the creation of artificial intelligence: Mary poppins 3000s of the world unite! AAAI Workshop: Human Implications of Human-Robot Interaction.

Wittgenstein, L. (1953). Philosophical investigations. Macmillan Publishing Company. 